\title{
On the Aging Behaviors of the Magnetic Stress Sensitivity of Very Fine Mo-Permalloy Ribbons for Memory Elements*
}

\author{
By Yukio Ichinose**, Masaru Kobayashi** \\ and Satoshi Taniguchi**
}

\begin{abstract}
The aging behaviors of the magnetic properties, especially of the magnetic stress sensitivity $S$, were investigated on the very fine Mo-permalloy ribbons finally cold-rolled from the fine wires.

$S$ always increases with aging and sometimes changes from the negative to the positive sign but such changes in $S$ are completely suppressed if ribbons are held at sub-zero temperature. On the aging process of $S$, three stages are observed in the temperature range between room temperature (R.T.) and $250^{\circ} \mathrm{C}$ : the first stage occurs at R.T. $\sim 100^{\circ} \mathrm{C}$, the second at $100^{\circ} \sim 200^{\circ} \mathrm{C}$ and the third at $200^{\circ} \sim 250^{\circ} \mathrm{C}$, their activation energies being evaluated as $0.95,1.5$ and $2.2 \mathrm{eV}$, respectively. It can be thought from these values that the aging process for each stage is mainly caused by the migration and extinction process of vacancy clusters for the first stage, by the same process of single vacancies for the second and by the rearrangement of dislocations for the third, respectively. Thus, such aging behaviors of $S$ can be interpreted in terms of the occurrence of the $\mathbf{K}$-state (short range order) peculiar to Mo-permalloys. It can be ascertained, therefore, that $S$ of the ribbons is sensitively influenced by working and aging.
\end{abstract}

(Received, March 30, 1968)

\section{Introduction}

On cold-deforming of metals or alloys, excess imperfections are introduced. In such a case, vacancies and interstitials migrate and disappear into the sinks such as dislocations or grain boundaries at relatively

* Originally published in Japanese in Journal of the Japan Institute of Metals, 32 (1968), 190.

** Central Research Laboratory, Hitachi Ltd., Tokyo, Japan. low temperatures. On these processings, atom diffusion and its subsequent rearrangement occur for the stress-relief. Such changes in the substructures naturally influence the mechanical and electrical properties of metals or alloys and are also recognized as aging behaviors.

As for the "twistor" memory(1), very fine Mo-

(1) A.H. Bobeck : Bell System Tech. J., 36 (1957), 1319. 
permalloy ribbons are utilized and these are prepared finally by cold-rolling the fine wires(2)(3). Such ribbons are very unstable in substructures and therefore the aging behaviors of the magnetic properties, similar to those of the mechanical and electrical ones, is possible to occur even near room temperature (R.T.). Actually, in the previous paper ${ }^{(4)}$, the magnetic stress sensitivity $S$ (or the stress sensitivity of anisotropy field $d H_{k} / d_{\sigma}$ ) of the ribbons was found to be aged at R.T. or a little higher temperature. There have, however, been no detailed investigations on the magnetic aging behaviors of Mo-permalloys.

The present study was carried out to examine the details of the aging behaviors of the magnetic properties, especially of the magnetic stress sensitivity $S$, for the very fine Mo-permalloy ribbons.

\section{Experiments and Discussions on the Magnetic Aging}

Among many working processes of ribbons for twistors*, the cold-drawing (process $R_{2}$ ) after the strand annealing (process SHT) is presumed to greatly influence the aging behavior of $S$ of the ribbons, the situation of which is similar to the final cold-rolling because of the considerably high reduction rate. Therefore, for the aging behaviors of the magnetic properties of the ribbons, the processing conditions of the fine wires in process $R_{2}$ and the alloy composition have to be taken into consideration.

In the following, the aging behaviors of the magnetic properties, especially of $S$, of the ribbons will be first examined and their dependence on the working processes and alloy compositions is investigated. Here, the ribbon samples are of about $7.5 \mu \times 120 \mu$ in cross section and were prepared finally by cold-rolling very fine Mo-permalloy wires of $35 \mu$ in diameter.

\section{Preparatory experiments on the aging at R.T. $\sim 75^{\circ} \mathrm{G}$}

At first, to examine the magnetic aging behaviors accompanied by the migration and extinction process of the imperfections introduced into the ribbons by cold-rolling, ribbon No. 584 was especially selected. This ribbon was cold-rolled from the fine wire pre-age at R.T. for $1000 \mathrm{hr}$ which is enough for the ribbon to be released or stabilized from the stress. Therefore, the aging observed in the ribbon may be thought to be caused by only the imperfections introduced in the cold-rolling process. Here, ribbon No. 584 is a Mopermalloy containing $4 \%$ Mo and 79.5\% Ni (by weight) and the aging temperatures are R.T. $\left(\simeq 25^{\circ} \mathrm{C}\right), 50^{\circ} \mathrm{C}$, $75^{\circ} \mathrm{C}$ and occasionally sub-zero temperature $\left(\simeq-70^{\circ} \mathrm{C}\right)$. The measurements of the aging behavior of $S$ (magnetic stress sensitivity), $H c$ (coercive force) and $u V_{1}$ (switching output) were started immediately after coldrolling. For one of the ribbons the effect of holding

(2) J. T. Ballenger : Western Elec. Eng., 4 (1960), 2.

(3) P. H. Mayer : Western Elec. Eng., 9 (1965), 2.

(4) Y. Ichinose, S. Taniguchi and M. Kobayashi : Trans. JIM, 9 (1968), 282

* The details on the working processes of ribbons for twistors were described in the previous paper(4). at "sub-zero" (sub-zero holding) on the aging behaviors of the magnetic properties was examined. "Subzero" means that the ribbon is put into dry ice immediately after cold-rolling. The measurements were in all cases carried out at R.T. after the ribbon was taken out.

The experimental results are shown in Fig. 1. It is found that on aging $\mathrm{Hc}$ tends to decrease and $u V_{1}$ and $S$ to increase. In more details, regular changes can be found in $S$ but not in $H c$ and $u V_{1}$, probably due to the reason that the measuring senstivity is very high in $S$ and low in $H c$ and $u V_{1}$. It is also seen in Fig. 1 that the relaxation curves of $S$ vs. aging time shift to the left side with increasing aging temperature, and that with sub-zero holding any changes in $S$ are not recognized throughout this experiment. If the ribbon is, however, taken out from sub-zero temperature to R.T. and successively held at R.T. (R.T. holding), then the relaxation eurve of $S$ vs. aging time agrees quite well with that of the ribbon for which the aging measurement was started immediately after cold-rolling. It is evident that the sub-zero holding suppresses completely the aging of the magnetic properties; this takes place only in subzero holding.

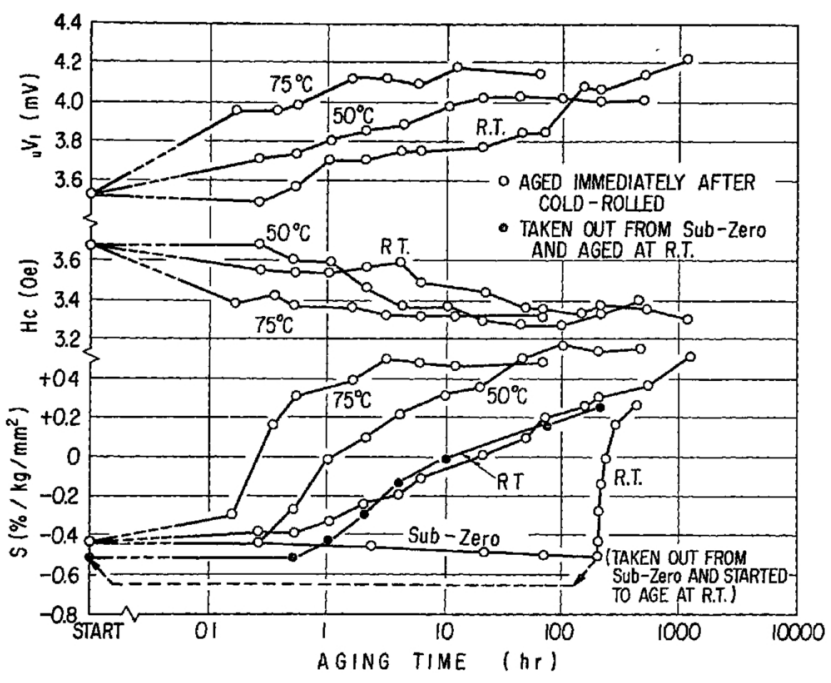

Fig. 1 Aging processes in the magnetic properties, the magnetic stress sensitivity $(S)$, the coercive force (Hc) and the switching output $\left(u V_{1}\right)$, of the finally cold-rolled Mo-permalloy ribbon (Sample : ribbon No. 584)

Furthermore, beside the above-mentioned coldrolling process, the processing conditions in the fine wires (process $R_{2}$ ) before cold-rolling are of great importance to the aging behaviors of $S$ in ribbons. Here, ribbon No. 641 was selected for experiments. This ribbon is the same as No. 584 except that it was prepared by cold-rolling the fine wire subjected to subzero holding until cold-rolling.

The experimental results are shown in Fig. 2, in which the results of ribbon No. 584 are included for comparison. It is found that $S$ is remarkably dependent on the processing conditions in process $A_{1}$. That is, $S$ of ribbon No. 584 is about $-0.4\left(\% / \mathrm{kg} / \mathrm{mm}^{2}\right)$ 


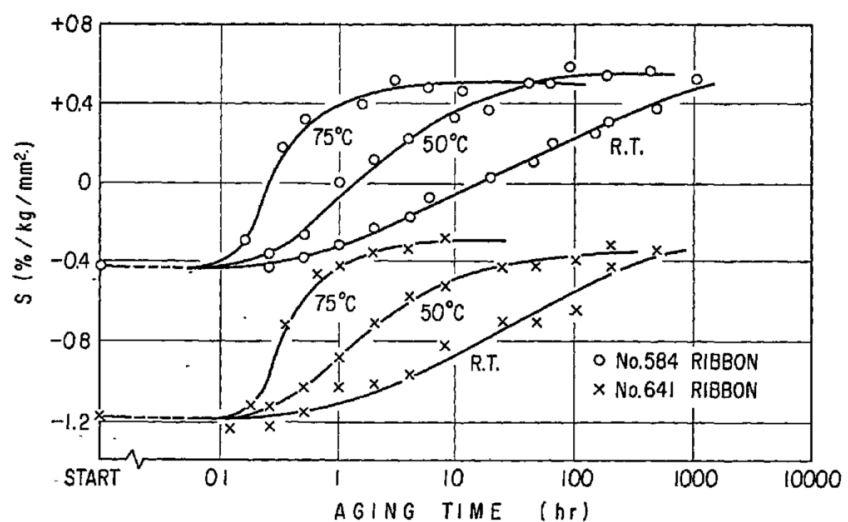

Fig. 2 Comparison of the aging processes in $S$ of the Mopermalloy ribbons cold-rolled from the long-aged and sub-zero held fine wires (ribbon No. 584 was long aged and ribbon No. 641 was subjected to sub-zero holding)

immediately after cold-rolling and about +0.6 after aging, while $S$ of ribbon No. 641 is about -1.2 at first and about -0.2 at last. From this it is seen that the amounts of change of $S$ due to aging are the same for the two ribbons but those individual values are quite different from each other. The $S$-value is usually larger in ribbon No. 584 than in ribbon No. 641, and after aging, $S$ of the former changes from the negative to the positive sign while $S$ of the latter remains in the negative sign.

\section{Experiments on the aging at R.T. $\sim 250^{\circ} \mathrm{G}$}

In the previous section, the aging behaviors of $S$ were preparatorily observed in the temperature range near R.T. F Further, this will be investigated in a wider temperature range between R.T. and $250^{\circ} \mathrm{C}$, using ribbon No. 641. The experimental results are shown in Fig. 3. According to this figure, three stages are observed on the $S$ vs. aging time curves. The first stage occurs at R.T. $\sim 100^{\circ} \mathrm{C}$, the second at $100^{\circ} \sim$ $200^{\circ} \mathrm{C}$ and the third at $200^{\circ} \sim 250^{\circ} \mathrm{C}$, respectively. Then, it is found that the $S$ stays within the negative sign below $100^{\circ} \mathrm{C}$ but changes to the positive sign over that temperature.

Further, to consider the mechanism of the aging

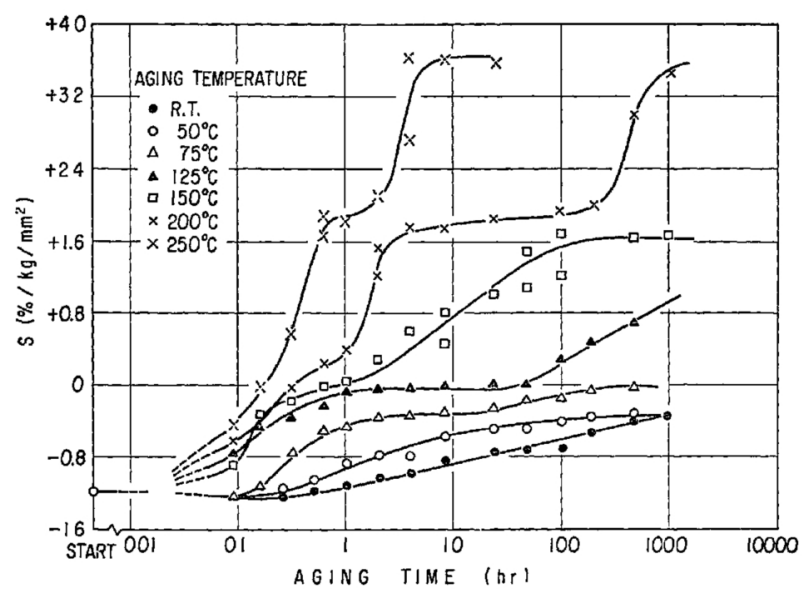

Fig. 3 Aging behaviors of $S$ of the Mo-permalloy ribbon in the temperature range between room temperature (R.T.) and $250^{\circ} \mathrm{C}$ (Sample: ribbon No. 64l)

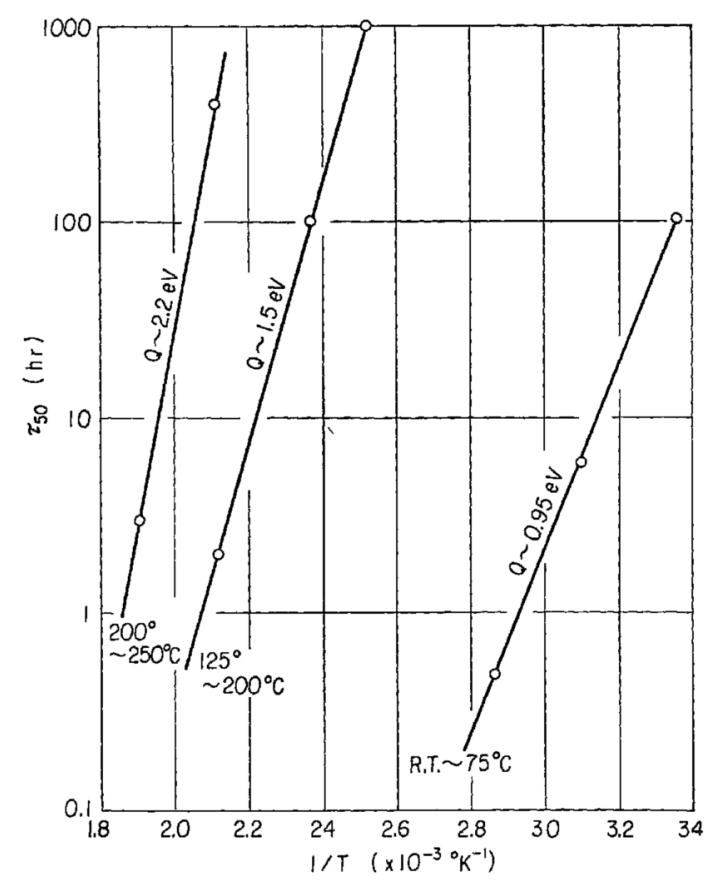

Fig. 4 Relations between the relaxation time $\left(\tau_{50}\right)$ and the reciprocal of the absolute aging temperature $(1 / T)$

Table 1 Activation energies (Q) evaluated from Fig. 4 and their comparison with the other workers' results

\begin{tabular}{|c|c|c|c|c|}
\hline \multirow{2}{*}{ Investigator } & \multicolumn{3}{|c|}{$\begin{array}{c}\text { Activation energy } Q \\
(\mathrm{eV})\end{array}$} & \multirow{2}{*}{$\begin{array}{l}\text { Measuring } \\
\text { Temp. range }\end{array}$} \\
\hline & $\begin{array}{l}\text { 1st } \\
\text { stage }\end{array}$ & $\begin{array}{l}\text { 2nd } \\
\text { stage }\end{array}$ & $\begin{array}{l}\text { 3rd } \\
\text { stage }\end{array}$ & \\
\hline This & 0.95 & 1.5 & 2.2 & R.T. $\sim 250^{\circ} \mathrm{C}$ \\
\hline Dolmatova et al. & 0.9 & 1.5 & - & R.T. $\sim 400^{\circ} \mathrm{C}$ \\
\hline Tolman & - & 1.6 & 2.2 & $119^{\circ} \sim 300^{\circ} \mathrm{C}$ \\
\hline Odani et al. & - & 1.6 & 2.3 & $100^{\circ} \sim 500^{\circ} \mathrm{C}$ \\
\hline
\end{tabular}

behavior of $S$, the relaxation time $\left(\tau_{50}\right)$ corresponding to the $50 \%$ change of $S$ in each stage is logarithmically plotted in Fig. 4 as a function of the reciprocal of absolute aging temperature (1/T). That is, the relations between $\log \tau_{50}$ and $1 / T$ are approximately linear and obey Arrhenius' law

$$
\tau_{50}=\tau_{0} \exp (Q / R T) \quad(\mathrm{hr}),
$$

where $\tau_{0}$ is the relaxation time coefficient, $Q$ the activation energy and $R$ the gas constant. The activation energies for the three stages in the $S$ vs. aging time curves are evaluated by the above equation to be $0.95 \mathrm{eV}$ for the first stage, $1.5 \mathrm{eV}$ for the second and $2.2 \mathrm{eV}$ for the third, respectively. These results are shown in Table 1 as compared with data of other workers. It is found that the present results agree quite well with those given by the other workers, that is, with Dolmatova et al.(5) for the first stage, with Dolmatova et al.(5) and Tolman ${ }^{(6)}$ for the second and with Tolman ${ }^{(6)}$ and Odani et al.(7) for the third

(5) R.P. Dolmatova et al. : Fiz. metal. metalloved., 19 (1965), 257.

(6) E. M.' Tolman : J. Appl. Phys., 31 (1961), 360S.

(7) Y. Odani et al. : Ordinary Meeting of Japan Inst. Metals at Tokyo (April, 1966). 
respectively. For the mechanism of the aging behavior of $S$, it may be thought from these magnitudes of the activation energies that the first stage is caused by the migration and extinction process of vacancy clusters (di- or tri-vacancies) ${ }^{(5)}$, the second by the same process of single vacancies ${ }^{(5)(6)}$ and the third by the rearrangement of dislocations (poligonization) $)^{(6)}$.

Meanwhile, according to the above observations, it is found that $S$ of the ribbon used for the present experiment changes from the negative to the positive sign with increasing aging temperature. This means that the alloy composition given $S \simeq 0$ is variable with the processing conditions of the ribbons. To ascertain this prediction, the temperature dependence of $S$ on isochronal aging was investigated in the Mo-permalloy ribbons containing $79 \sim 81 \% \mathrm{Ni}$ prepared according to the working processes of the twistors.

The experimental results are shown in Fig. 5. It is found that $S$ stays within the negative sign for all ribbons as cold-rolled, but that after aging it rapidly increases and at last takes the positive sign in the low $\mathrm{Ni}$ ribbons while it hardly changes in the high $\mathrm{Ni}$ ribbons. Consequently, the alloy composition of $S \simeq 0$ is evidently found to shift to the Ni-rich side by aging. Also, this fact possibly shows that the magnetostriction $\lambda_{s}$ changes with the change in $S$ by aging ${ }^{(4)}$.

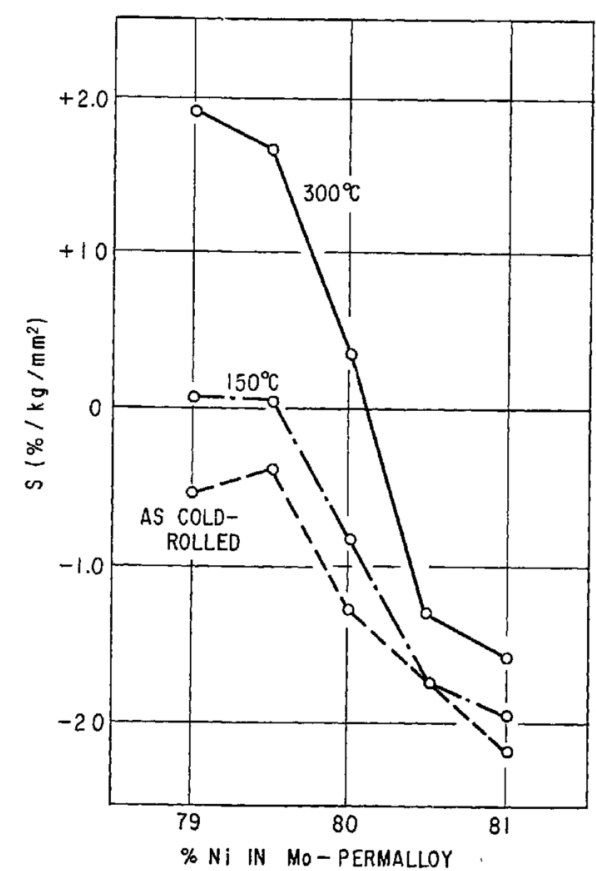

Fig. $5 \mathrm{Ni}$ content dependence of $S$ in isochronal aging

\section{Summary of the Experimental Results and Discussions on the Depen- dence of $S$ on Working Processes}

From the experimental results obtained hitherto (together with the results in the previous paper), it becomes clear that $S$ is remarkably dependent on the working history of ribbons such as the strand annealling (process SHT), the cold reduction of fine wires (process $R_{2}$ ), and the aging of fine wires and ribbons (processes $A_{1}$ and $A_{2}$ ). These anomalous phenomena observed in $S$ are schematically illustrated in Fig. 6 , where figure (a) shows the decrease of $S$ with the cold reduction rate in process $R_{2}$, (b) the complicated behavior of $S$ with the aging temperature in process SHT, (c) the increase of $S$ and occasional changes of its sign from negative to positive by aging in processes $A_{1}$ and $A_{2}$, and (d) the shift of the alloy composition giving $S \simeq 0$ to the Ni-rich side with increasing aging temperature. These facts give us very important imformation that $\lambda_{s}$ like $S$ is sensitively dependent on the working prócesses of the ribbons and changes from the negative to the positive sign or from the positive the negative sign as a result of some changes in substructures. This prediction is supported by the experimental results in the previous paper. That is, it was apparent that $S$ and $d H_{k} / d \sigma$ of ribbons were both smaller than zero just after cold-rolling, nearly zero after aging at R.T. for $24 \mathrm{hr}$ and larger than zero after aging at $250^{\circ} \mathrm{C}$ for $1.5 \mathrm{hr}$. This evidence shows that $\lambda_{s}$ like $S$ changes from the negative to the positive sign by aging since $d H_{k} / d \sigma \propto \lambda_{s}$ theoretically. Here, the amounts of change in $\lambda_{s}$ are at most smaller than about $10^{-7}$; this order of magnitude seems to be at variance provided the changes in substructures related to the K-state peculiar to Mo-permalloy(5)(8) (10) are induced by the migration and extinction process of excess imperfections such as vacancies and dislocations. In this case the cold-working promotes the occurrence of the disorder state and the aging treatment the order state. In Fig. 7, the compositional dependence of the magnetostriction in the order or disorder state given by Bozorth(11) is shown, from which it is predicted that $\lambda_{s}$ changes its sign in the

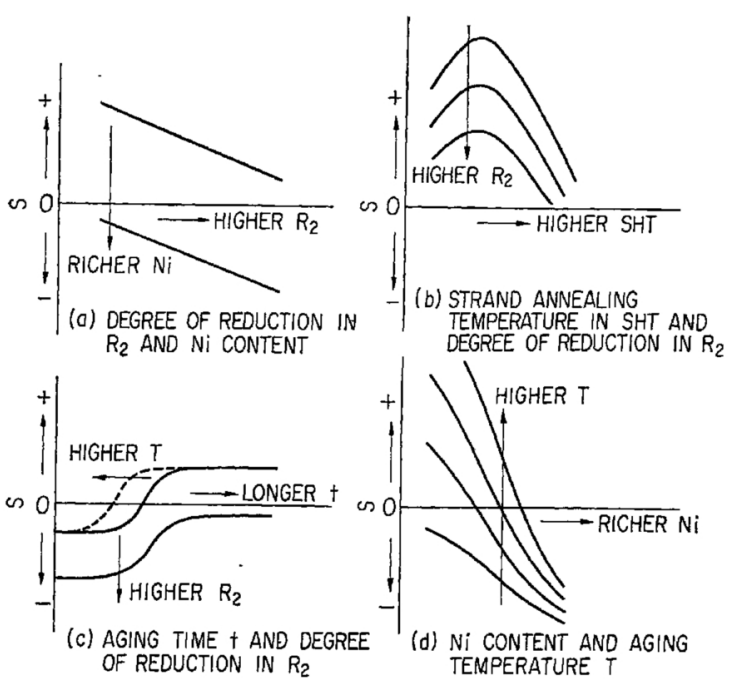

Fig. 6 Schematic illustrations of the anomalous phenomena found out in $S$ of the cold-rolled Mo-permalloy ribbons

(8) F. Assmus and F. Pfeiter : Z. Metallk., 42 (1951), 294.

(9) F. Pfeifer and I. Pfeifer : Z. Metallk., 55 (1964), 398.

(10) M. A. Bol'shanina and A. D. Korotayev : Fiz. metal. metalloved., 17 (1964), 512.

(11) R.M. Bozorth and J.G. Walker: Phys. Rev., 89 (1953), 624. 


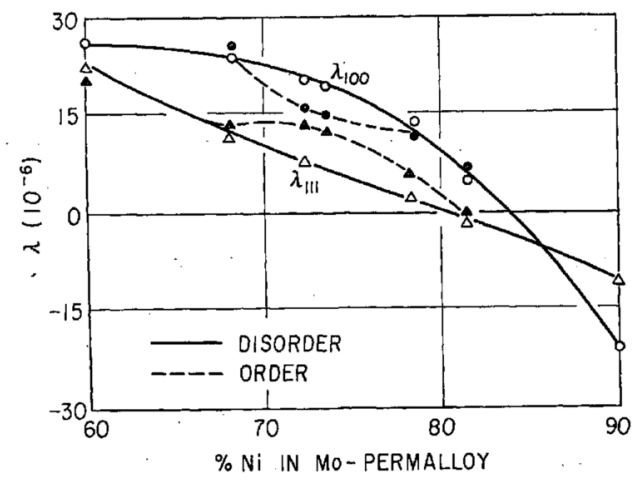

Fig. $7 \mathrm{Ni}$ content dependence of the magnetostriction in Fe-Ni binary system (After R.M. Bozorth and J.G. Walker ${ }^{(11)}$ )

alloy composition near Mo-permalloy with substructural transition between the disorder and order states. In what follows, the $\mathrm{K}$-state will be investigated from measurements of the electrical resistivity of fine wires and ribbons.

As is well known, Mo-permalloy alloys show excellent magnetic properties if furnace-cooled at some optimum rate after annealing. The reason is that both the magnetocrystalline anisotropy constant $K_{1}$ and the magnetostriction constant $\lambda_{s}$ become nearly zero due to the formation of the optimum order state $^{(12)}$.

Such changes in the magnetic properties have been recently interpreted in relation to the $\mathrm{K}$-state (short range order), the features of which are phenomenally represented as the decrease of the electrical resitivity by cold-deforming and its increase by aging after quenching and cold-deformation(13). This will be then examined experimentally from measurements of the electrical resistivity of fine wires and ribbons in association with their processing conditions such as the cold reduction and the heat treatment. Here, the experimental samples are the same ribbons as used for the above experiments and the fine wires before cold-rolling with the degree of reduction of 0 (as full-annealed), 50, 73.5 and 90\%, respectively.

The experimental results are shown in Figs. $8 \sim 10$. Fig. 8 shows the change of the electrical resistivity as a function of the cold reduction rate in the fine wires. The electrical resistivity is found to decrease with the cold reduction rate, which may be caused by the transition of order $\rightarrow$ disorder in the K-state. Figs. 9 and 10 are the electrical resistivity vs. aging temperature curves for the ribbons and wires, respectively. It is found that the electrical resistivity initially increases and attains a peak at $420^{\circ} \mathrm{C}$ and then again decreases over that temperature; further, it attains a valley at $700^{\circ} \mathrm{C}$ and again increases over that temperature. The $420^{\circ} \mathrm{C}$ peak may be caused by the transition of disorder $\rightarrow$ order $\rightarrow$ disorder in the K-state, where the disorder $\rightarrow$ order transition brings about the increase in electrical resistivity, and the order $\rightarrow$ disorder transition the decrease in electrical resistivity and at the complete order the peak reveals itself. The

(12) K. Aoyagi : Japanese J. Appl. Phys., 4 (1965), 551.

(13) T. Nagashima : Bul. Japan Inst. Metals, 3 (1964), 281.

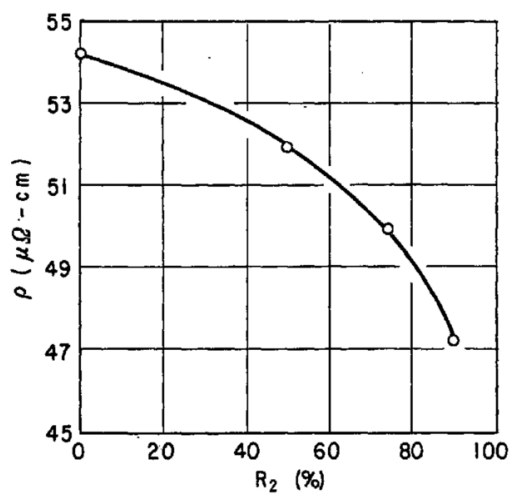

Fig. 8. Dependence of the specific resistivity $(\rho)$ on the cold reduction rate $\left(R_{2}\right)$ in the fine Mo-permalloy wires

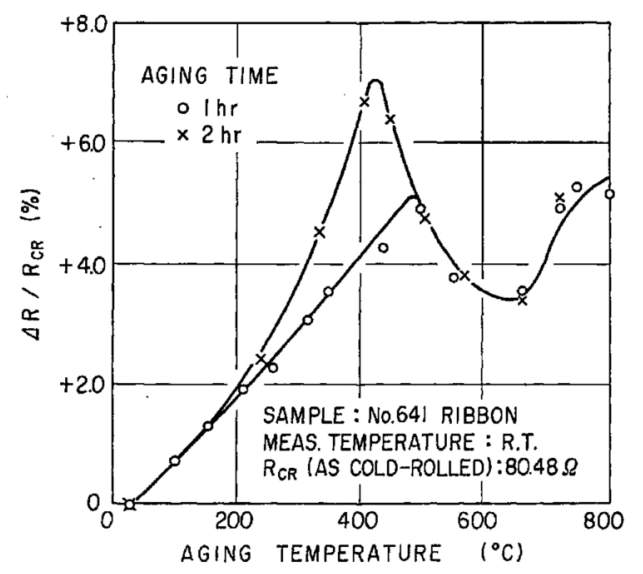

Fig. 9 Dependence of the electrical resistivity on the aging temperature in the cold-rolled Mo-permalloy ribbons

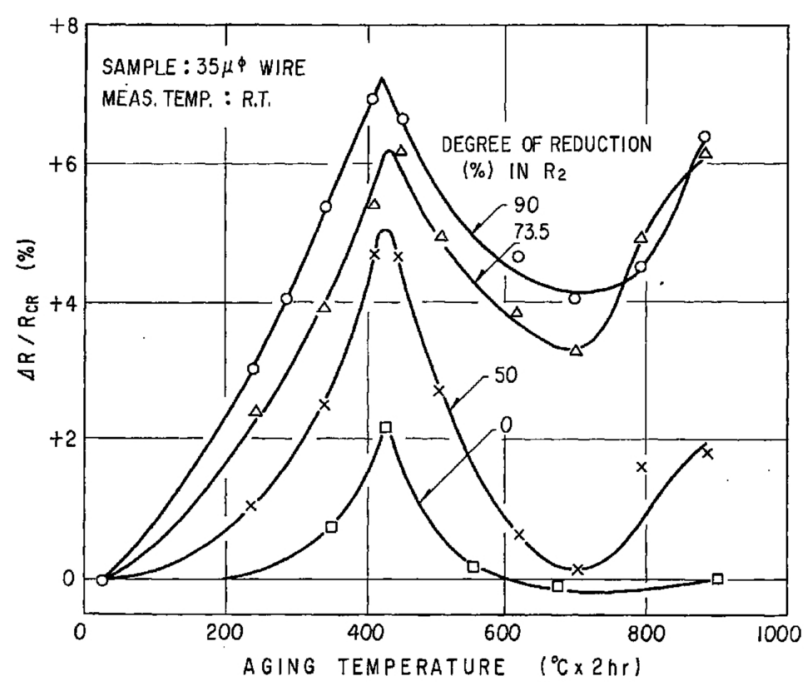

Fig. 10 Dependence of the electrical resistivity on the aging temperature and the cold reduction rate in the fine Mo-permalloy wires

increase of the electrical resistivity over $700^{\circ} \mathrm{C}$ may be attributed to the formation of the K-state through the atomic rearrangement due to rapid diffusion of the thermally excited excess vacancies during cooling ${ }^{(14)}$.

From these observations, it is evidently convinced that the very fine Mo-permalloy ribbons and wires are in the very unstable disorder state immediately

(14) J. Takamura : Acta Met., 6 (1961), 547. 
after cold-deformation and so that they are inclined to transform to a more stable order state even at R.T. or a little higher. Such changes in the $K$-state (disorder $\leftrightarrows$ order transition) have been well known to influence the magnetic properties such as magnetic anisotropy and magnetostriction.

From the above considerations, the anomalous phenomena in $S$ as shown in Fig. 6 can be clearly interpreted as being caused by the substructural changes related to the order $\underset{\leftarrow}{ }$ disorder transition in the K-state. That is, the decrease in $S$ of the ribbons with the cold reduction is accompanied with the decrease in $\lambda_{\mathrm{s}}$ through the order $\rightarrow$ disorder transition in the $\mathrm{K}$-state, while the increase in $S$ by aging is accompanied by the increase in $\lambda_{5}$ through the disorder $\rightarrow$ order transition in the K-state. Here, it was occasionally seen that the sign of $S$ changes from the negative to the positive sign. This fact is of great importance, since it implies that the similar change occurs in the sign of $\lambda_{\mathrm{s}}$ due to the disorder $\rightarrow$ order transition in the $\mathrm{K}$-state. Also, from comparison with the anomalies in the electrical resistivity, it can be understood that the complicated behaviors of $S$ to the strand annealing temperature are closely related to the changes of the $\mathrm{K}$-state.

\section{Conclusion}

Of the magnetic properties of the very fine Mopermalloy ribbons finally cold-rolled from the fine wires, the aging behaviors of the magnetic stress sensi- tivity $S$ were investigated. The experimental results are summarized as follows;

(1) By aging at relatively low temperatures, $S$ always increases and sometimes changes its sign from minus to plus, but such changes of $S$ are completely suppressed by sub-zero holding.

(2) On the aging process of $S$, three stages are observed in the temperature range between room temperature (R.T.) and $250^{\circ} \mathrm{C}$ : the first stage occurs below $100^{\circ} \mathrm{C}$, the second at $100^{\circ} \sim 200^{\circ} \mathrm{C}$ and the third at $200^{\circ} \sim 250^{\circ} \mathrm{C}$, activation energies being evaluated as $0.95,1.5$ and $2.2 \mathrm{eV}$, respectively. It is predicted from these magnitudes that the aging process for each stage is mainly caused by the migration and extinction process of vacancy clusters for the first stage, by the same process of single vacancies for the second and by the rearrangment of dislocations for the third, respectively.

(3) The anomalous phenomena observed in $S$ by cold-working and aging are quite consistent with the anomalies in the electrical resistivity, closely related to the order $\leftrightarrows$ disorder transition in the $K$-state peculiar to Mo-permalloy alloys. Therefore, $S$ is sensitively dependent on working and aging because of the changes in the K-state.

\section{Acknowledgments}

The authors wish to thank Mr. S. Hishiyama and Mr. T. Yamashita of the Central Research Laboratory, Hitachi Ltd., for their helpful assistance to this work. 